\title{
Self-Diffusion of Tungsten on Some Facets of Tungsten Microcrystal
}

\author{
T. BIERnAT* AND R. B£ASZCZYSZYN \\ Institute of Experimental Physics, University of Wrocław \\ pl. Maxa Borna 9, 50-204 Wrocław, Poland
}

The field emission current fluctuation method was applied to investigate surface self-diffusion of tungsten on the (311) and (711) regions (along the zone line (211)-(100)) of a tungsten microcrystal (in the form of the field emitter tip of radius about $300 \mathrm{~nm}$ ) in the temperature range 500-850 K. The surface diffusion parameters were obtained from spectral analysis of the current fluctuations. The results are discussed from the aspects of the substrate structure.

PACS numbers: 79.70.+q, 68.90.+g, 05.40.-a

\section{Introduction}

Self-diffusion process plays an essential role in the disordering of surface and as a consequence in the roughening, melting and faceting of the crystal surface [1]. One of the methods of detection of the surface disorder via the changes in surface self-diffusion is the field emission (FE) current fluctuation (field emission flicker noise) method initiated by Kleint in 1960 [2] and widely developed by Gomer [3] and others [4]. Gomer and co-workers studied in detail the surface self-diffusion on the chosen regions in the zones (011)-(112) and (011)-(001) of the field emitter tip in the form of a tungsten microcrystal applying Gomer's theoretical approach to the analysis of experimental autocorrelation functions $[5,3,6]$, which allowed them to determine the self-diffusion parameters: temperature dependences of the surface diffusion coefficient $D$, activation energy $E$ and self-diffusion constant $D_{0}$. Depending on the substrate structure and temperature one- or two-dimensional (1D or $2 \mathrm{D})$ diffusion has been postulated from the analysis of the shape of the correlation function [5].

In this paper the self-diffusion was examined on two regions $\mathrm{W}(311)$ and $\mathrm{W}(711)$ situated on the zone line (211)-(100) of the tungsten microcrystal via analysis of the measured FE current fluctuations performed in terms of spectral density function by applying the Gesley and Swanson theory [7].

*corresponding author; e-mail: teresa@ifd.uni.wroc.pl 
The collective (multiatomic) self-diffusion studies widened the single atom diffusion measurements $[8,9]$ giving a more complete inside into the diffusion phenomena.

\section{Experimental details}

The experimental system and procedure was similar to that presented in Ref. [10]. The measurements of FE current fluctuations were carried out under UHV conditions (pressure below $1 \times 10^{-10}$ Torr) with the sealed-off glass FE microscope of probe-hole type. The emitter tip temperature was stabilised and controlled using the temperature dependence of the resistance of an emitter-loop segment with potential leads. The FE current from a circular probed region of approximately $10 \mathrm{~nm}$ radius was directed to the Faraday collector by an external magnetic field. After amplification of the probe-hole current, its ac component was processed by an analog-digital converter PC card. The sampling frequency was $20 \mathrm{kHz}$. The spectral analysis of the FE current fluctuations from the investigated regions was made.

\section{Results and discussion}

The regions chosen for the study of self-diffusion are presented in Fig. 1.

\subsection{Spectral density functions}

The spectral density functions $S(f)$ of FE fluctuations were determined as a function of temperature. The experimental results are compared with the theoretical spectral density function derived by Gesley and Swanson [7]. Those authors have assumed that the FE current fluctuations are caused by fluctuations in the number of adparticles on the small probed area and obtained, for an unbounded 2D diffusion, the spectral density function $S\left(u_{\mathrm{p}}\right)$ described by equation

$$
S\left(u_{\mathrm{p}}\right)=C_{\mathrm{FN}}\left\langle(\delta N)^{2}\right\rangle r_{\mathrm{p}}^{2} D^{-1} T\left(u_{\mathrm{p}}\right),
$$

where $u_{\mathrm{p}}=2 \pi f r_{\mathrm{p}}^{2} D^{-1}$ is the normalised frequency, $T\left(u_{\mathrm{p}}\right)$ is an expression composed of the Kelvin functions, $\left\langle(\delta N)^{2}\right\rangle$ is the mean-square fluctuation of the number of adparticles on the probed area with radius $r_{\mathrm{p}}, D$ is the surface diffusion coefficient, $C_{\mathrm{FN}}$ is the Fowler-Nordheim term. The diffusion coefficient $D$ was obtained by comparison of the abscissas of theoretical and experimental curves.

Figure 2 shows the experimental spectral density function $S\left(u_{\mathrm{p}}\right)$ and the theoretical one (smooth line) fitted to the experimental values. The shape of the spectral density functions suggests the occurrence of $2 \mathrm{D}$ self-diffusion for the $\mathrm{W}(311)$ and $\mathrm{W}(711)$ regions in the temperature ranges $630-850 \mathrm{~K}$ and $500-680 \mathrm{~K}$, respectively. For the $\mathrm{W}(311)$ below $600 \mathrm{~K}$, the fitting of the theoretical $S(f)$ to experimental points is not possible. In this case the $1 \mathrm{D}$ or pre-diffusion processes can be considered. It can be noticed that for the $\mathrm{W}(711)$ facet the temperature range of $2 \mathrm{D}$ self-diffusion is lower than that for the W(311). Beyond the investigated temperature range probably a new process (of roughening) occurs. 


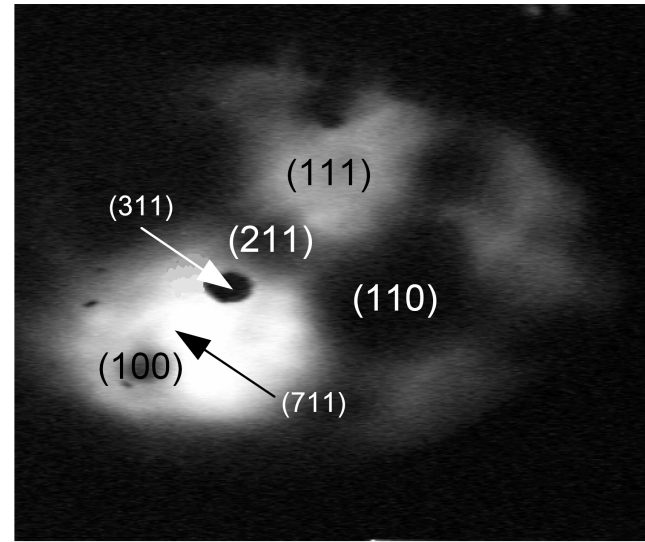

Fig. 1. Field emission pattern of the clean tungsten surface with some microcrystal facets marked. The collector probe-hole is visible as the dark spot covering the (311) facet. Arrows indicate the investigated regions.

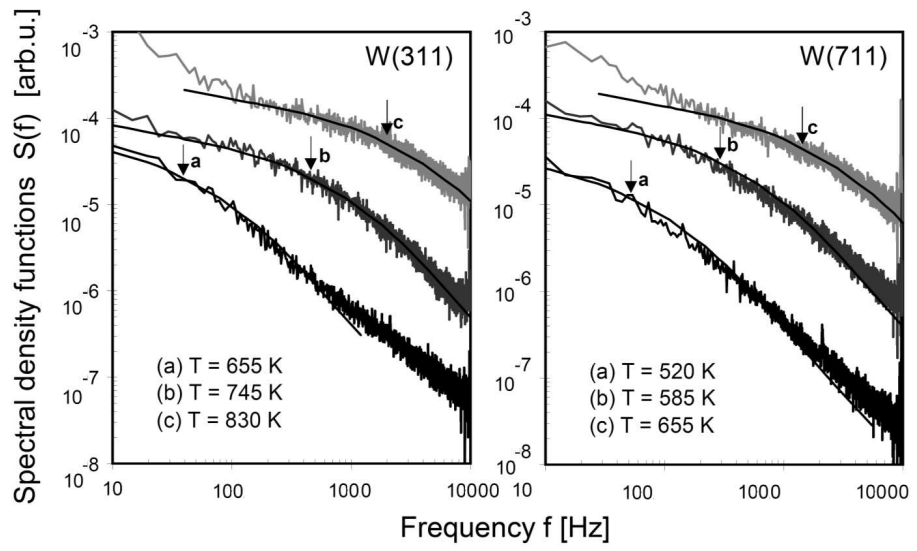

Fig. 2. Typical behaviour of the $S(f)$ functions with increasing temperature. Smooth lines represent the theoretical spectral density function $S\left(u_{\mathrm{p}}\right)$ fitted to the experimental values. Arrows indicate the experimental frequencies corresponding to the normalised frequency $u_{\mathrm{p}}=1$ (curves are shifted in the vertical direction by one decade for better viewing).

\subsection{Activation energy of surface self-diffusion}

As it was shown for the first time by Swanson [11] and then in papers [5, 6] at moderate temperature the FE current fluctuations can be seen on clean metal surfaces, arising from the creation and motion of detached atoms and other defects.

A comparison of the theoretical spectrum with the experimental $S(f)$ 's presented in Fig. 2 shows that the latter can be described by the diffusion model only in a restricted frequency range, i.e. in part of the measured band, varying with temperature. Particularly the "low frequency part" at higher temperature cannot 
be described by the path diffusion model, applied in the Gesley and Swanson paper [7]. Obviously, different processes have to be taken into account in the case of complex spectra, especially for non-isotropic regions like the bcc $\mathrm{W}(311)$ and (711) facets. The appearance of critical fluctuations of an order-disorder phase transition, the presence of traps on the plane of interest subject to surface diffusion, interactions between adatoms and also the domain-wall motion might be considered. The different current fluctuation sources and the models to describe them are discussed in Refs. [5, 6, 12].

Assuming that surface diffusion plays a dominant role in the FE current fluctuations in the temperature range of interest, the surface diffusion coefficient $D$ was determined. The activation energy $E$ for surface diffusion and the pre-exponential factor $D_{0}$ were calculated from the relation $D=D_{0} \exp (-E / k T)$. The results are presented in Fig. 3. The self-diffusion activation energy for the $\mathrm{W}(311)$ facet $(E=1.02 \mathrm{eV} /$ atom $)$ is higher than that for the $\mathrm{W}(711)$ facet $(E=$ $0.69 \mathrm{eV} /$ atom).

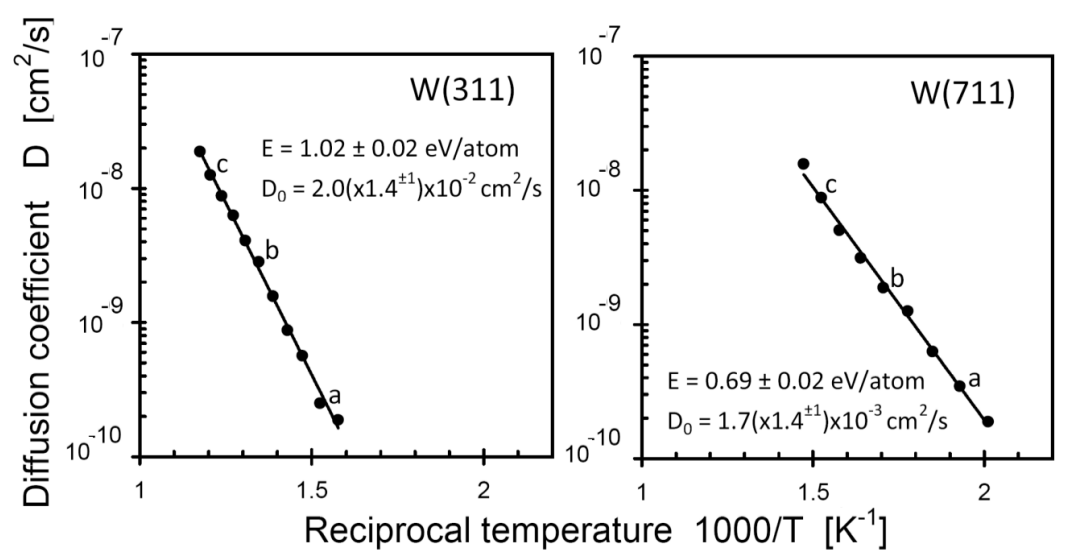

Fig. 3. Temperature dependence of the diffusion coefficient. Points $a-c$ correspond to curves in Fig. 2.

The regions (311) and (711) chosen for investigation lie in the zone line (211)(100) (the angle between the (211) and (100) poles is $35.26^{\circ}$ ). The angle between the (211) and (311) poles is $10.02^{\circ}$ whereas that between (211) and (711) poles is $23.84^{\circ}$. The (311) region consists of terrace fragments of (211)-orientation and the step edges of (110) structure running in the [011] direction. Next, the terraces of (711) region (at $11.42^{\circ}$ from the (100) pole) correspond to the structure of (100) surface and the step edges are parallel to the [011] direction. Ball models of the bcc W(311) and (711) facets are presented in Fig. 4.

According to the Gesley and Swanson theory [7], the shape of the experimental spectral density function suggests the $2 \mathrm{D}$ diffusion. Taking into account the structure of these substrates, the diffusion picture seems to be rather clear. 


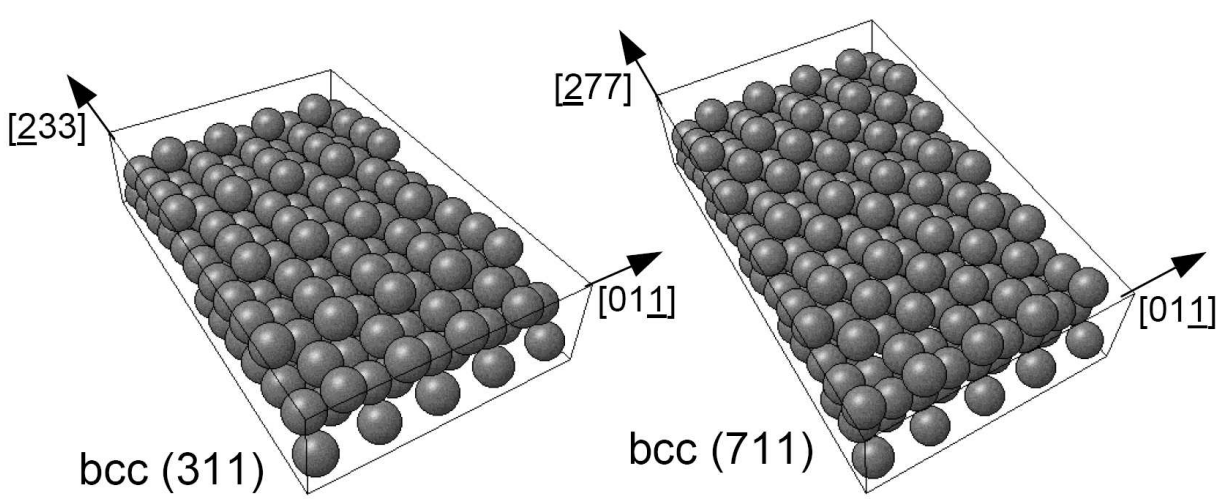

Fig. 4. Ball model of the investigated regions (adapted after K. Hermann, Fritz-Haber Institute, Berlin).

For the (311) region, at a higher temperature the detached atoms, mainly from the edges of the terraces and from the atom rows of $\langle 111\rangle$ direction, diffuse along the $\langle 111\rangle$ direction and perpendicular to the through direction [011], jumping over the step edges and atom rows. The (711) region represents an atomically smoother substrate in relation to the (311) and thus the value of activation energy is lower than that for the (311) region.

In Table results for the collective (multiatomic) and single $\mathrm{W}$-atom self-diffusion on chosen regions of $\mathrm{W}$ microcrystal, determined by the field ion and field emission current fluctuation method, are presented. The results of this paper are in good agreement with the others for the (211) region presented in Table, although scatter of data depending on method and temperature range is noticeable.

TABLE

Diffusion parameters of $\mathrm{W} / \mathrm{W}$ along the zone line (211)-(100).

\begin{tabular}{c|c|c|c|c}
\hline \hline Region & $\begin{array}{c}\text { Temperature } \\
\text { range }[\mathrm{K}]\end{array}$ & $\begin{array}{c}\text { Activation energy } \\
{[\mathrm{eV} / \text { atom }]}\end{array}$ & $\begin{array}{c}\text { Prefactor } D_{0} \\
{\left[\mathrm{~cm}^{2} / \mathrm{s}\right]}\end{array}$ & Ref. \\
\hline$(211)$ & $500-740$ & 0.56 & $5 \times 10^{-7}$ & {$[5]$} \\
along channels & $500-826$ & 0.73 & $3 \times 10^{-5}$ & {$[6]$} \\
across channels & $526-752$ & 0.29 & $4.2 \times 10^{-9}$ & {$[6]$} \\
& $752-910$ & 1.04 & $5 \times 10^{-4}$ & {$[6]$} \\
single atom diffusion & $260-325$ & 0.81 & $3.41 \times 10^{-3}$ & {$[9]$} \\
\hline$(311)$ & $630-850$ & 1.02 & $2 \times 10^{-2}$ & this paper \\
\hline$(711)$ & $500-680$ & 0.69 & $1.7 \times 10^{-3}$ & this paper
\end{tabular}

As it results from the diffusion theory, the prefactor $D_{0}=f d^{2}$, where $f$ denotes the attempt frequency and $d$ is the jump length, if the change of entropy 
is neglected [3]. The distance between the atom rows on the $\mathrm{W}(211)$ plane is $0.447 \mathrm{~nm}$ and that between the $\mathrm{W}$ atoms along the $\langle 111\rangle$ directions 0.274 . Usually it is assumed that $f=10^{12} \mathrm{~s}^{-1}$, then in our case we obtain the distances $d=$ $1.4 \mathrm{~nm}$ and $0.8 \mathrm{~nm}$ for the diffusion on the (311) and (711) regions, respectively. According to the result of Antczak and Ehrlich [8] it should be assumed that in this temperature range the diffusion occurs via a long distance jumping mechanism which requires a higher activation energy for diffusion. The determined values for $d$ indicate that this diffusion mechanism is feasible although the jump length at the distance of $1.4 \mathrm{~nm}$ on the (311) region seems to be little reasonable.

In this method an effect of the high electric field (about 30-40 MV/cm) on the diffusion makes a serious problem. This was discussed in papers $[5,6]$ with the conclusion that the effect is rather weak; the experiments were carried out under conditions for which the build-up effect could be neglected [13].

\section{Acknowledgments}

Helpful discussion with Dr. S. Surma and Dr. G. Antczak are gratefully acknowledged.

\section{References}

[1] E.H. Conrad, Prog. Surf. Sci. 39, 65 (1992).

[2] Ch. Kleint, Surf. Sci. 200, 472 (1988).

[3] R. Gomer, Rep. Prog. Phys. 53, 917 (1990).

[4] R. Błaszczyszyn, Prog. Surf. Sci. 42, 117 (1993).

[5] Y.M. Gong, R. Gomer, J. Chem. Phys. 88, 1359 (1988); ibidem, p. 1370.

[6] D.-S. Choi, R. Gomer, Surf. Sci. 230, 277 (1990).

[7] M.A. Gesley, L.W. Swanson, Phys. Rev. B 12, 7703 (1985).

[8] G. Antczak, G. Ehrlich, Surf. Sci. Rep. 62, 39 (2007).

[9] G. Antczak, Phys. Rev. B 73, 1 (2006).

[10] T. Biernat, R. Błaszczyszyn, Appl. Surf. Sci. 230, 81 (2004).

[11] L.W. Swanson, Surf. Sci. 70, 165 (1978).

[12] Ch. Kleint, Surf. Sci. 25, 394 (1971).

[13] P.C. Bettler, F.M. Charbonnier, Phys. Rev. 119, 85 (1960). 